\begin{tabular}{|c|c|c|c|c|c|}
\hline Revista Clío América & ISSN: 1909-941X & Vol. 12 & No. 23 & enero - junio de 2018 & $8-24$ \\
\hline
\end{tabular}

\title{
La conciencia tributaria en el proceso de recaudación de impuestos en Santa Elena
}

\author{
Tax awareness in the tax collection process in Santa Elena
}

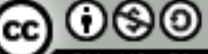 BY Ne SA}

RESUMEN: La recaudación tributaria en la provincia de Santa Elena ha tenido un incremento paulatino durante los últimos años debido a una adecuada administración de la gestión de cobro por parte del ente controlador (Servicio de Rentas Internas) y a las reformas implementadas en los últimos tiempos; sin embargo, el monto recaudado por las sanciones aplicadas mantuvo una tendencia al aumento. Adicionalmente, se encuentra el nivel de preparación de la ciudadanía en materia tributaria, lo que promueve un análisis de las acciones de instrucción que han sido ofrecidas por el Servicio de Rentas Internas (SRI) y conlleva a aseverar que la capacitación tributaria no llega al ciudadano común debido a que las capacitaciones in situ se realizan en los principales cantones de las provincias de Ecuador. La investigación analiza la incidencia de la conciencia tributaria en la recaudación de los impuestos de la provincia de Santa Elena a partir de una metodología cualitativa con enfoque exploratorio-descriptivo y propone una guía metodológica para crear formadores en materia de tributación en asociación con gremios de la localidad como estrategia para lograr un mejoramiento en la toma de decisiones, contribuyendo a la formación y al desarrollo de la conciencia tributaria ciudadana en esta comunidad.

Palabras clave: sistema tributario - conciencia tributaria - capacitación - impuestos - multas.

JEL: H25, H26, H71

ABSTRACT: In recent years, tax collection in the province Santa Elena has had a gradual increase, due to proper administration of the collection management by the entity of control - Internal Revenue Service (SRI) - and reforms implemented in recent time. However, the amount raised by the sanctions maintained a rising trend. In addition, is the level of preparedness of citizens in tax matters, which promotes an analysis of training activities that have been offered by the Internal Revenue Service and assert that tax training does not reach the ordinary citizen because the in Situ trainings are held in the main districts of the provinces of Ecuador. The research analyzes the incidence of tax awareness in the collection of taxes in the province of Santa Elena, from a qualitative methodology and a exploratorydescriptive approach, and proposes a methodological guide to train educators on tax issues in partnership with unions of the town, as a strategy to achieve an improvement in decision making about issues encountered in the community and to contribute to the formation and development of public awareness, important training for all those who decide to start a business.

Keywords: Tax System - Tax Awareness - Financial Education - Taxes - Fines.

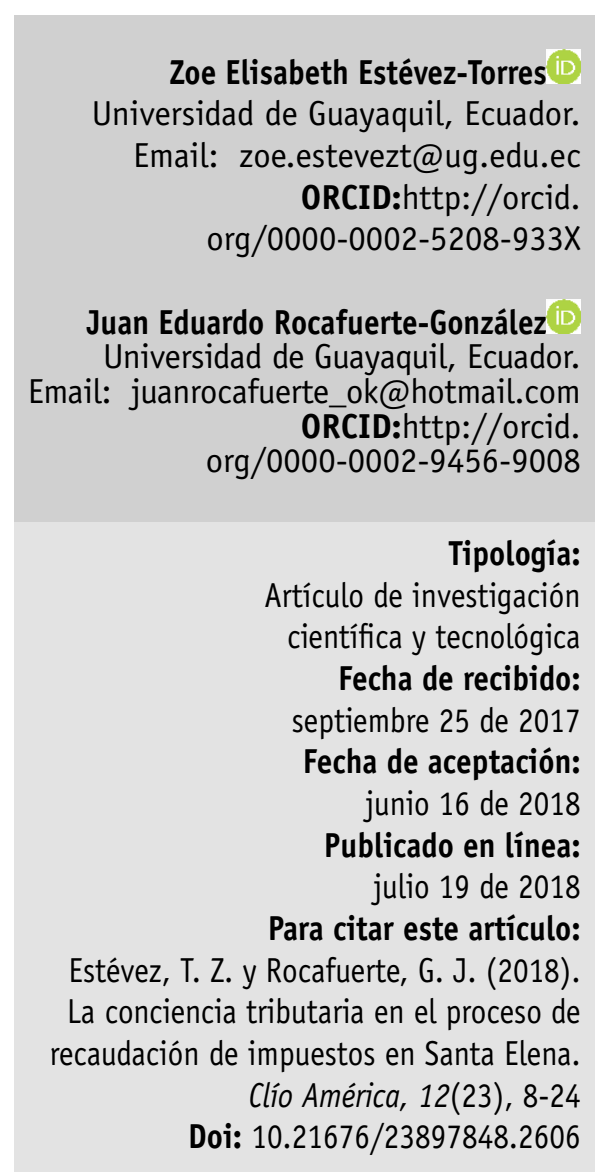




\section{INTRODUCCIÓN}

Los tributos constituyen un medio efectivo utilizado por el Estado para la recaudación de fondos destinados a cubrir las necesidades sociales colectivas. Dado que la gestión tributaria efectiva cobra vital importancia en la agenda del ente superior encargado de regir el sistema tributario, es importante generar conciencia en favor de la necesidad de su recaudación, a partir de la capacidad de convocatoria y la preparación en el tema tributario de la población.

De acuerdo a los datos reflejados en los resultados del censo realizado por el Instituto Nacional de Estadísticas y Censo INEC (2010), la población de Santa Elena es de 308693 habitantes y se encuentra concentrada principalmente en edades jóvenes. La Población Económicamente Activa (PEA) está representada por el 35,28 \% de la población. Mientras la mayor parte de la población masculina tiene una ocupación artesanal, las mujeres se dedican por lo general al comercio.

Según este organismo, la mayor parte de la población no aporta o no está afiliada al seguro social. La escolaridad de la población mayor de 24 años es de 8,9 años aprobados para las mujeres y de 8,7 años aprobados para los hombres, para un valor medio de 8,8. El analfabetismo asciende al 5,2\% en la población mayor a 15 años.

En la península de Santa Elena aún se manifiestan deficiencias relacionadas con la recaudación de impuestos, bien por el registro erróneo de información al momento de la declaración, bien por la presentación de la información a destiempo. Esta situación provoca que gran parte de los contribuyentes acudan a pagar el servicio de declaración de impuestos a terceros, siendo la causa principal de este hecho la carencia de conocimiento tributario $\mathrm{y}$, además, la falta de ética del contribuyente, demostrada en la evasión del impuesto por parte de algunas personas que declaran ingresos cero o utilizan facturas vencidas engañando, a su vez, al consumidor.

La investigación realizada por Leon, Martinez, Zavala y Espinel (2010) destaca "el bajo nivel educacional de la población de la provincia de Santa Elena como el determinante principal de la situación de pobreza existente, recomendando ampliar la cobertura de la educación media por ser el sector de mayor necesidad de atención" (p. 9). Así, se entiende la importancia de la formación no solo para el desarrollo personal, sino también para el crecimiento económico.

En su investigación, Washco (2015) hace énfasis en el nuevo modelo tributario adoptado por Ecuador en el período 2009-2013 y prevé afectaciones a las recaudaciones producto del desconocimiento, la falta de difusión, la displicencia de los contribuyentes y los constantes cambios en las normas, lo que repercute en una probable disminución de las transacciones generadoras del hecho tributario ya que estos factores fomentan indirectamente el comercio informal. Sostiene, dentro de su investigación, una potencial disminución en el cumplimiento de las obligaciones tributarias de los contribuyentes en el período sustentado a causa de un incremento de las recaudaciones de interés por mora y multas tributarias, aun cuando la recaudación sigue un patrón de crecimiento constante.

Por su parte, Jaramillo y Aucanshala (2013) sostienen que deben aplicarse estrategias que permitan concientizar a los contribuyentes acerca de la importancia de cubrir sus obligaciones impositivas, así como que es responsabilidad del ente recaudador alentar a los contribuyentes a efectuarlas.

La recaudación de los impuestos depende de la conciencia tributaria de los contribuyentes, discernimiento que se forma e incentiva a través de capacitaciones constantes del ente regulador. En ocasiones, circunstancias como la situación geográfica, los pocos recursos técnicos, humanos y financieros para ejercer estas inducciones no permiten a la entidad llegar a las comunas de la península, donde convergen un número importante de artesanos y comerciantes minoristas.

Lo expuesto anteriormente conduce a preguntarse acerca de la incidencia de la conciencia tributaria de los contribuyentes en el proceso de recaudación de impuestos en la provincia de Santa Elena. Para 
responder a esto es necesario analizar los factores que influyen en la conciencia tributaria. Para ello, se definen las siguientes acciones:

- Analizar las variaciones de la recaudación tributaria en la provincia de Santa Elena en el periodo 2011-2015 a través de cuadros comparativos individuales y consolidados.

- Examinar la posible incidencia de las multas en las fluctuaciones presentadas en las recaudaciones tributarias del período objeto de análisis.

- Proponer alternativas para lograr una recaudación tributaria efectiva.

La utilidad de la investigación recae sobre el conocimiento objetivo de la incidencia de la conciencia tributaria en el proceso de recaudación de impuestos en la provincia de Santa Elena, siendo una fuente de información para determinar las fortalezas y debilidades en la gestión recaudadora del Servicio de Rentas Internas en la provincia y permitiendo, a su vez, emitir por parte del investigador recomendaciones válidas para la mejora de la gestión anteriormente descrita.

El estudio concibe demostrar la importancia y la necesidad de que los contribuyentes conozcan los métodos tanto técnicos como manuales para pagar sus tributos para que, de esta forma, se contribuya con los aportes al estado; estos se utilizan para la construcción de obras en beneficio propio y de la comunidad.

Los aspectos que otorgan relevancia a la investigación están relacionados con la complementariedad del análisis de datos estadísticos de las contravenciones aplicadas en la recaudación tributaria con la aplicación de un cuestionario para evaluar el grado de conocimiento de la población acerca de los términos tributarios, permitiendo determinar la incidencia de la conciencia tributaria en la recaudación de los impuestos.

Las limitaciones de la investigación estuvieron determinadas por la falta de información relacionada con las medidas locales adoptadas por los organismos estatales encargados por ley de realizar las capacitaciones a los pobladores de la provincia de Santa Elena.

\section{METODOLOGÍA}

Se utiliza un enfoque mixto o cuali-cuantitativo de tipo descriptivo (teniendo en cuenta que se espera describir la conciencia tributaria de los ciudadanos de la provincia de Santa Elena) y explicativo (para exponer el impacto que la misma produce en el sector impositivo tributario durante el período 20112015, marcado por grandes reformas tributarias).

Se abordan métodos tanto teóricos como empíricos para el desarrollo del trabajo. Dentro de los métodos teóricos, se aborda el inductivo-deductivo para ir de lo general a lo particular y viceversa, lo que permite estudiar el comportamiento del sistema tributario en la provincia de Santa Elena desde un todo hacia sus partes, contrarrestar los resultados y llegar a conclusiones. En cuanto a los métodos empíricos, se utilizan la revisión bibliográfica para compilar los datos estadísticos de los tributos, las multas aplicadas durante el período de estudio y la encuesta como instrumento de campo con el objetivo de conocer el conocimiento tributario de los contribuyentes de la zona, específicamente artesanos y comerciantes, así como de develar si en algún momento habían recibido capacitaciones tributarias por parte del Servicio de Rentas Internas. Se ha tomado como referencia la información estadística del Servicio de Rentas Internas.

El universo considerado para la aplicación del instrumento de campo está constituido por la población económicamente activa de la provincia de Santa Elena, representada por 108043 habitantes. Aplicando la fórmula (1) correspondiente a un muestreo probabilístico, y considerando una población conocida $(\mathrm{N})$, una proporción de éxito $(\mathrm{p})$ del $50 \%$ para asegurar que en la población hay la máxima diversidad posible, un nivel de confianza (Z) del 90 $\%$ y un margen de error (e) del $10 \%$ dado que según el SRI, no toda la PEA está afiliada al seguro social y, por consiguiente, no toda aporta al presupuesto del estado, se obtuvo una muestra (n) de 68 personas.

$$
n=Z^{2 *} p(1-p) /\left(1+\left(Z^{2 *} p(1-p)\right) / e^{2} N\right)
$$




\section{Cuestiones introductorias al Sistema Tributario en Ecuador}

En relación al desarrollo de la investigación, es preciso determinar el concepto de "impuestos". Troya (como se citó en Maldonado, 2009) se refiere a estos como:

Aquellos tributos exigidos por el Estado en virtud de su potestad de imperio, sin que exista una contraprestación y que el contribuyente se ve obligado a pagar por encontrarse en el caso previsto por la disposición legal que configura la existencia del tributo (p. 17).

Andino et al (2012) abordan un concepto de impuestos que va más allá del sufragio a la actividad estatal, considerándole como "un vínculo palpable entre los ciudadanos y el Estado, y entre las personas que conforman la comunidad o sociedad", por lo cual lo reconoce como una "contribución solidaria" que explica de la siguiente forma:

Por el lado del financiamiento, el conjunto de tributos debe ser equitativo para que sea justo; ello debe complementarse con el uso que se hace de los recaudos. Y por el lado de la contribución los impuestos se convierten en elementos que representan un sentimiento de pertenencia a un grupo humano con objetivos comunes $y$, a la vez, son mecanismos que otorgan a sus pagadores el derecho y la obligación de velar por el destino de esos recursos (p. 40).

Sarmiento (2015) destaca que "el tributo tiene como finalidad esencial posibilitar la financiación del gasto público, aunque puede dirigirse también a satisfacer otros objetivos públicos como por ejemplo propiciar la creación de empleo, estimular el desarrollo económico entre otros" (p. 31).

Vicente de la Casa (2012) refiere que la antigua Ley General Tributaria española define el término "impuestos" como "los tributos exigidos sin contraprestación cuyo hecho imponible está constituido por negocios, actos o hechos de naturaleza jurídica o económica que ponen de manifiesto la capacidad económica del contribuyente" (p.154).
Cortés (como se citó en Vicente de la Casa, 2012) manifiesta que "han de contribuir a las cargas públicas quienes tengan capacidad económica para ello"(p.151), destacando que el soporte de la obligación tributaria reside en la existencia de un valor económico en poder de los individuos para hacer frente al pago del tributo luego de deducir sus gastos.

Sainz de Bujanda (como se citó en Novoa, 2006) acota al respecto que:

El principio de capacidad contributiva actúa, sobre todo, dentro de la tributación como sistema, de tal suerte que la adecuación de los hechos imponibles a la capacidad contributiva se produce, primordialmente, a través de la conexión de unos hechos con otros y de la carga total que generan para los sujetos que lo realizan.(p.104)

En opinión de Masbernat Muñoz (2010) “el impuesto debe graduarse atendiendo a la capacidad individual de pago, y no sólo porque el obligado es un ser capaz de obtener rendimientos" (p.326)

Asimismo, Giullianni Fonrouge (como se citó en Novoa, 2006) asevera que "en el orden de los conceptos jurídicos, el poder tributario no conoce otros limitantes que los inherentes a la soberanía o poder de imposición del Estado"(p.104).

En todas las definiciones ofrecidas se destacan como características principales de los impuestos su importancia como fuente de recursos para sostener el gasto público; de allí su obligatoriedad. Esto, sin obviar la racionalidad en correspondencia con los ingresos individuales y su propósito fundamental: el goce y provecho de toda una sociedad.

A la relación de los impuestos se le denomina "Sistema Tributario". A continuación, se exponen conceptos de diferentes autores sobre el tema:

Para Valdivia (2010) "consiste en la fijación, cobro y administración de los impuestos y derechos internos y los derivados del comercio exterior que se recaudan en el país, además administra los servicios aduanales y de inspección fiscal" (p.1). 
Ferreiro (como se citó en Moreira, 2015) refiere que un sistema tributario es:

Un conjunto de gravámenes que están juntos y que deben aplicarse como partes integrantes de un todo, ya que cualquier grupo de impuestos que se establezcan y apliquen por un determinado poder se denomina sistema tributario; el cual, implica cierta coordinación de los diferentes impuestos entre sí con el sistema económico dominante y con los fines fiscales y extra fiscales de la imposición (p. 27).

El sistema tributario ecuatoriano abarca el conjunto de impuestos exigidos por ley; estos son administrados por el Servicio de Rentas Internas (SRI) y por otras entidades como municipios, gobiernos autónomos descentralizados, etc. Este sistema busca procurar que la estructura impositiva planteada y definida para el país permita promover la equidad y justicia social a través de preceptos como los del "Buen Vivir".

De acuerdo con la opinión de Peláez y Gutiérrez (2016), "los principales impuestos a considerar en Ecuador son el Impuesto al Valor Agregado, el Impuesto a la Renta y el Impuesto a los Consumos Especiales"(p.4). Cabezas (2010) refiere que el primero

Grava valor añadido en cada fase del proceso de producción y distribución y se genera el momento de realizar la transacción de bienes y servicios. Siendo considerado como un impuesto regresivo ya que la tasa que se paga por este impuesto es la misma independientemente del nivel de ingresos de las personas que compran el producto 0 adquieren el servicio. (pp. 15-16)

Acerca del segundo expresa que "grava los ingresos 0 rentas de las personas, por las actividades laborales o económicas, una vez descontados los gastos deducibles y las utilidades de las empresas", considerándole como un impuesto progresivo que grava con una mayor tasa impositiva a quienes obtienen mayores rentas. Para el tercero comenta que "es un impuesto sobre consumos específicos, que grava ciertos bienes y servicios nacionales o importados, detallados en el artículo 82 de la Ley de Régimen Tributario Interno". Desde el punto de vista económico, también es considerado un impuesto regresivo.
La carta magna hace referencia a la esencia y alcance de estos impuestos en su Artículo 300:

Art.300.- El régimen tributario se regirá por los principios de generalidad, progresividad, eficiencia, simplicidad administrativa, irretroactividad, equidad, transparencia y suficiencia recaudatoria. Se priorizarán los impuestos directos y progresivos. La política tributaria promoverá la redistribución y estimulará el empleo, la producción de bienes y servicios, y conductas ecológicas, sociales y económicas responsables (Constitución de la República del Ecuador, 2008).

Sin embargo, y como refiere Jiménez (2015), "en ocasiones resulta difícil para el contribuyente entender los impuestos, y las normas que los regulan a menudo no contribuyen a reducir su complejidad" (p. 131).

En opinión de Giulliani Fonrouge, (como se citó en Del Busto Vargas, 2014) "el cobro de un impuesto, sin ley que lo autorice, es un despojo y viola el derecho de propiedad" (pp.31-32). Es así como la Ley de Creacion del Servicio de Rentas Internas (1997) determina el inicio de las actividades del SRI el 1 de enero de 1998, destacando entre sus funciones "ejecutar la política tributaria aprobada por el Presidente de la República y efectuar la determinación, recaudación y control de los tributos internos del Estado". Según refleja el diario El Telégrafo en su artículo «La recaudación se triplicó entre 2007 y 2013» (2014), su surgimiento se fundamenta en "la alta evasión, alimentada por la ausencia de cultura tributaria y la corrupción existente entre varios funcionarios recaudadores de impuestos de esa época".

A partir del año 2007, con el triunfo del economista Rafael Correa Delgado como presidente de la república, una de las prioridades del nuevo gobierno fue la de preparar el camino para la implementación de herramientas que permitieran asegurar la equidad en el país; entre estas, sobresale la elaboración y preparación de una nueva constitución a través de la Asamblea Nacional de Ecuador que, en lo referente a temas tributarios, señala en su artículo número 135 la posibilidad de que sea el poder ejecutivo quien 
pueda establecer, modificar o eliminar impuestos cuando expresa que "solo la Presidenta o Presidente de la República podrá presentar proyectos de ley que creen, modifiquen o supriman impuestos, aumenten el gasto público o modifiquen la división político administrativa del país" (Constitución de la República del Ecuador, 2008, p. 53).

Desde el 2007 hasta el 2013 se realizaron una serie de cambios que dotaron de justicia al sistema y recuperaron su rol regulador y promotor del desarrollo, creando impuestos extra fiscales que corrigen conductas sociales, ambientales o económicamente nocivas para la sociedad - que no tienen una finalidad recaudatoria sino que buscan desincentivar determinadas conductas-. Todo esto es producto del mandato constitucional: "promover impuestos directos y progresivos [...] y promover conductas sociales, económicas y ambientales responsables" (Jiménez, 2015, p. 111).

El Servicio de Rentas Internas y su página web constituyen una plataforma al servicio de la ciudadanía para cualquier orientación en el ámbito de las obligaciones tributarias. Asimismo, en la actualidad, los distintos colegios existentes en el país y las universidades incluyen en sus programas el control contable y tributario con el fin de promover conductas sociales responsables y que disminuyan los actos de fraude, evasión y elusión fiscal. Balseca (2012) así lo recoge cuando destaca:

El fortalecimiento de la conciencia tributaria, como parte de un proceso de transformación social, constituye una buena parte de la misión institucional del Servicio de Rentas Internas, orientada a promover y exigir el cumplimiento de las obligaciones tributarias en el marco de principios éticos y legales, para asegurar una efectiva recaudación que fomente la cohesión social. Pero siendo importante el papel de la administración como motor de cambios, la transformación social nunca sería posible sin el convencimiento y esfuerzo responsable y participativo de organizaciones representativas, colectivos y personas individuales en la construcción de un régimen de Buen Vivir (p. 127).

Castañeda (2015) razona al respecto de esta manera:
Un individuo altamente educado podría ser consciente de la importancia de la provisión pública [...] le sería más fácil aceptar su obligación de aportar a su financiamiento así como también es factible que esta misma persona conozca las oportunidades que presenta el sistema tributario para evadir $y$, eventualmente, lo haga. Pese a lo anterior, en la literatura empírica predominan los resultados consistentes con la idea de que el primer efecto compensa al segundo. Además, el papel de la educación puede ser más importante si el contribuyente está estudiando, pues ello supone una mayor probabilidad de que se cuestione su papel en la sociedad (p. 117).

Para Lozano y Tamayo (2016), "la gestión de la ética en las entidades públicas puede ayudar a prevenir la corrupción, evitando llegar a la sanción de las conductas no permitidas"(p.8). Así lo destaca Vargas (2014):

El Servicio de Rentas Internas cuenta con el Centro de Estudios Fiscales que fomenta la investigación y pensamiento del contexto tributario, económico y social en el departamento de Estudios, mientras que en el Departamento de Formación Tributaria y Fiscal se diseñan cursos y programas que desarrollan y fortalecen las competencias y el talento en los servidores públicos del SRI y ciudadanía en general a favor del fomento de la cultura tributaria (p. 79).

Con todo, la demanda para estos cursos es mucho mayor que la oferta que se brinda, principalmente por la no disponibilidad de la totalidad del talento humano necesarios, de los recursos técnicos y de los recursos financieros.

La coexistencia humana está regida por principios y reglas que marcan la conducta del hombre ante la sociedad, por lo que "ética" es un término amplio relacionado con el comportamiento y las acciones humanas. El propósito principal de la recaudación es el de redistribuir las riquezas dentro de la sociedad de forma planificada, consciente y objetiva. Por consiguiente, se necesita una base ética para su desarrollo; esto conduce a inferir que "ética" y "recaudación tributaria" son dos términos que deben ir de la mano.

Betancur (2010) entiende por "ética": 
la reflexión que se realiza sobre los actos humanos, en cuanto ellos comportan, de manera individual o colectiva, una orientación hacia la práctica del bien, o hacia la exclusión de éste que se entiende como la práctica del mal. Lo correcto (bien) y lo incorrecto (mal) se entenderán con referencia a la dignificación de los seres humanos y el cumplimiento de unos mínimos universales que permiten hacer juicios de valor acerca de las consecuencias que tienen las acciones personales y colectivas (corporativas) (p. 77).

Ziccardi (2016) considera que "la ética proporciona una serie de criterios y marcos teóricos indispensables para normar la conducta, haciendo al ser humano responsable y capaz de tomar decisiones a la luz de los valores universales". Por su parte, Lozano y Tamayo (2016) opinan que "la ética describe la orientación de un individuo en relación con un comportamiento bueno o malo, donde lo que es ético se alinea con lo primero y lo que no lo es, con lo segundo" (p. 8). Según Gilligan (como se citó en Fascioli, 2010), "el concepto central de la ética es la responsabilidad" (p. 44), entendida esta como el compromiso de transparencia con la sociedad.

Para Yañez (2015) "la evasión tributaria es una actividad ilegal, por medio de la cual los contribuyentes reducen el monto del impuesto que, al proceder de forma correcta, les habría correspondido pagar" (p. 173).

Para Lozano y Tamayo (2016) “la conexión entre las conductas legales elusivas y las conductas ilegales evasivas no representa per se una ruptura, sino una continuación provocada por las diferentes facetas legales e ilegales que se pueden adoptar al buscar minimizar la carga tributaria" (p. 5).

Todos los individuos deben contribuir con el Estado de acuerdo a su capacidad de pago. Se atenta directamente contra las finanzas públicas al evadir las obligaciones tributarias contraídas y al no ingresar la suma prevista o suprimir de la misma un beneficio indebido, transgrediendo la aplicación del principio de justicia fiscal en el reparto de los tributos y propiciando la generalización de una conducta deshonesta e irresponsable.
En la actualidad, el proceder de forma ética es un compromiso consigo mismo más allá del deber que implica para con la sociedad. Es una realidad que, en muchas ocasiones, los impuestos están determinados por normas y reglas un tanto complicadas, pero el desconocimiento no exime de la obligación ni, mucho menos, justifica el fabricar o falsificar los resultados.

Jiménez (2015) considera que "no es fácil para la mayoría de la población entender los impuestos, y las normas que los regulan a menudo no contribuyen a reducir su complejidad" (p. 131). Sin embargo, el desconocimiento, la inexperiencia o la ignorancia no justifican el incumplimiento de la ley ni instan a alterarla a conveniencia.

De acuerdo al Reglamento para la aplicación de incentivos a la producción y prevención del fraude fiscal (2015), se emplean un conjunto de sanciones agrupadas en tres categorías conforme a su impacto en la recaudación; se explican a continuación:

- La falta de presentación o presentación tardía de declaraciones patrimoniales.

- Las declaraciones tardías del impuesto a la renta, el impuesto al valor agregado (como agente de retención y percepción), las retenciones de ambos impuestos, el impuesto a consumos especiales y anexos también tendrán sanción, aunque se presenten en cero.

- Las contravenciones y faltas reglamentarias, dentro de las que se recogen: la no actualización del Registro Único de Contribuyentes (RUC); no tener un registro de ingresos y egresos; no comparecer ante las oficinas del Servicio de Rentas Internas o no exhibir documentación cuando la administración lo requiera; no entregar el comprobante de retención hasta cinco días después de recibido el comprobante de venta; emitir sin los correctos requisitos de llenado, facturas, notas de venta, liquidaciones, notas de crédito y débito; emitir liquidaciones de compra en casos no autorizados, entre otros. 
El Servicio de Rentas Internas (2014), en su Instructivo para la aplicación de sanciones pecuniarias, ha determinado las siguientes divisiones 0 estratos:

- Estrato 1: Contribuyentes especiales.

- Estrato 2: Sociedades con fines de lucro.

- Estrato 3: Personas naturales obligadas a llevar contabilidad.
- Estrato 4: Personas naturales no obligadas a llevar contabilidad y sociedades sin fin de lucro, determinando las diferentes cuantías que deben ser pagadas según el tipo de falta (p. 25).

A continuación, se exponen, en la Tabla 1, los valores de las cuantías establecidas de acuerdo a las clasificaciones.

\section{Tabla 1.}

\section{Clasificación de sanciones pecuniarias y su cuantía.}

\begin{tabular}{|c|c|c|c|c|c|c|c|c|c|}
\hline \multirow[b]{2}{*}{ CONTRIBUYENTE } & \multicolumn{3}{|c|}{ CONTRAVENCIÓN } & \multicolumn{3}{|c|}{ FALTA REGLAMENTARIA } & \multicolumn{3}{|c|}{ DECLARACIONES TARDÍAS } \\
\hline & Tipo A & Tipo B & Tipo C & Tipo A & Tipo B & Tipo C & $\begin{array}{c}\text { Antes de } \\
\text { proceso de } \\
\text { sanción }\end{array}$ & $\begin{array}{l}\text { Al inicio } \\
\text { de proceso } \\
\text { de sanción }\end{array}$ & $\begin{array}{l}\text { Al notificar } \\
\text { resolución } \\
\text { sanción }\end{array}$ \\
\hline Estrato 1 & 125,00 & 250,00 & 500,00 & 83,25 & 166,50 & 333,00 & 250,00 & 375,00 & 500,00 \\
\hline Estrato 2 & 62,50 & 125,00 & 250,00 & 41,61 & 83,25 & 166,50 & 125,00 & 187,50 & 250,00 \\
\hline Estrato 3 & 46,25 & 62,50 & 125,00 & 35,81 & 41,62 & 83,25 & 62,50 & 93,75 & 125,00 \\
\hline Estrato 4 & 30,00 & 46,25 & 62,50 & 30,00 & 35,81 & 41,62 & 31,25 & 46,88 & 62,50 \\
\hline
\end{tabular}

Fuente: elaboración propia basada en el Instructivo para la aplicación de sanciones pecuniarias del Servicio de Rentas Internas (2014).

\section{RESULTADOS}

\section{Evolución de la Recaudación Tributaria efectuadas por el Servicio de Rentas Internas en la provincia de Santa Elena durante el período 2011-2015}

La evolución de la recaudación tributaria efectuada por el Servicio de Rentas Internas en la provincia de Santa Elena durante el período 2011-2015 muestra un incremento sustancial en los dos primeros años, siendo la recaudación realizada en el año 2013 el monto de mayor significación: con relación al ejercicio económico anterior se presenta un aumento del $25 \%$ de las recaudaciones. Para el año 2014, la tendencia en recaudaciones se mantiene estable, pero no existe un incremento mayor al 2,99\% en términos generales. En el período se incrementó la recaudación en casi 8 millones de dólares. El análisis efectuado puede comprobarse a continuación en la Tabla 2. 


\section{Tabla 2.}

Evolución trimestral de la recaudación tributaria. Periodo 2011-2015.

\begin{tabular}{|l|c|c|c|c|c|}
\hline \multicolumn{1}{|c|}{ Meses } & Marzo & Junio & Septiembre & Diciembre & Total \\
\hline Año 2011 & 1274710,31 & 1527219,64 & 1673498,84 & 1150287,44 & 16885740,45 \\
\hline Año 2012 & 1758484,07 & 1388854,96 & 2046396,16 & 1956481,63 & 19255763,56 \\
\hline Año 2013 & 1714425,32 & 2656220,75 & 2526681,85 & 2009158,96 & 24206309,05 \\
\hline Año 2014 & 2567205,55 & 1916876,40 & 2255857,54 & 1915996,58 & 24929111,24 \\
\hline Año 2015 & 1892257,00 & 1702917,00 & 2072045,00 & 1782426,00 & 24674949,00 \\
\hline
\end{tabular}

Fuente: elaboración basada en los datos estadísticos obtenidos del SRI (2014).

Si bien entre el 2011 y 2014 las estadísticas indican un incremento en las recaudaciones, este no pasa por un aumento en el número de contribuyentes, sino por un aumento en la productividad de los contribuyentes ya registrados. Es importante recordar que, para el año 2013, los precios del petróleo superaron los 100 dólares, lo que generó cierta bonanza económica que derivó en seguridad para invertir, mayor flujo de circulante y apertura de mercados.
En lo que respecta al año 2015, se puede observar en la Figura 1 un decrecimiento en los montos de recaudación del 2,99\% puesto que, con el inicio de la debacle en los precios del petróleo, los buenos síntomas descritos en el párrafo anterior comenzaron a atenuarse debido a que la caída en los precios del ingreso número uno del país sugería la llegada de una recesión económica que traería consigo afectaciones de tipo socio económico como la inflación, el desempleo, el subempleo, etc., entre otras.

\section{Figura 1.}

Recaudación total porcentual.

\section{EVOLUCIÓN PORCENTUAL DE RECAUDACIÓN}

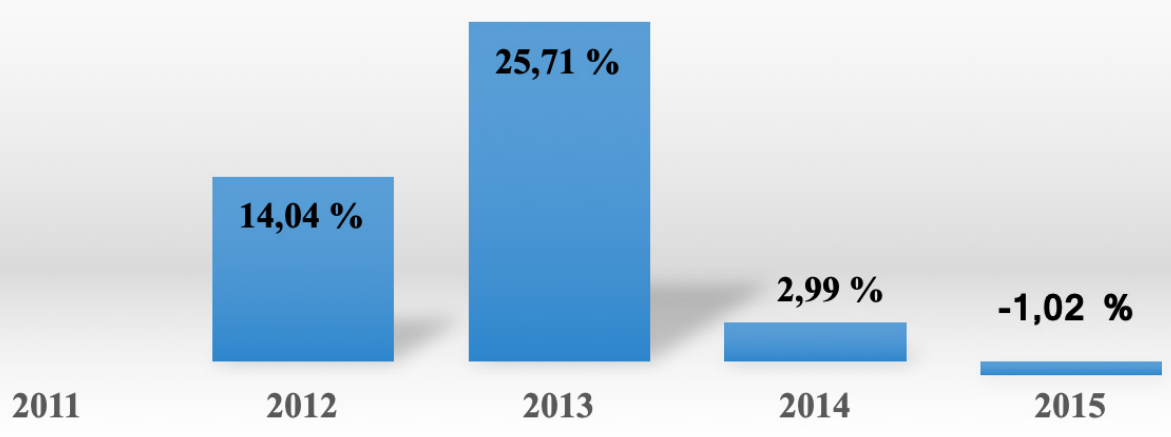

Fuente: elaboración propia basada en los datos estadísticos obtenidos del SRI (2014). 
Otra situación que amerita ser tomada en cuenta, a pesar de que se ha reiterado que no ha existido un descenso considerable en las recaudaciones tributarias en el 2015, pero sí un estancamiento y potencial decrecimiento, es el tema de la salvaguardias arancelarias aplicadas por el Gobierno Nacional en el mes de marzo de 2015; esta situación provocó la caída del interés de compra de los consumidores debido a la especulación y al aumento en los precios de cerca de 2800 bienes, a los que se les asignó salvaguardias.

De acuerdo a datos obtenidos del Servicio de Rentas Internas, el impuesto que tuvo mayor crecimiento entre los años 2011 y 2015 fue el pago por concepto del IVA mensual, tal y como se puede apreciar en la Figura 2.

\section{Figura 2.}

Recaudación IVA mensual período 2011-2015

\section{RECAUDACIÓN IVA MENSUAL 2011- 2015 \\ (en unidad de dólar)}

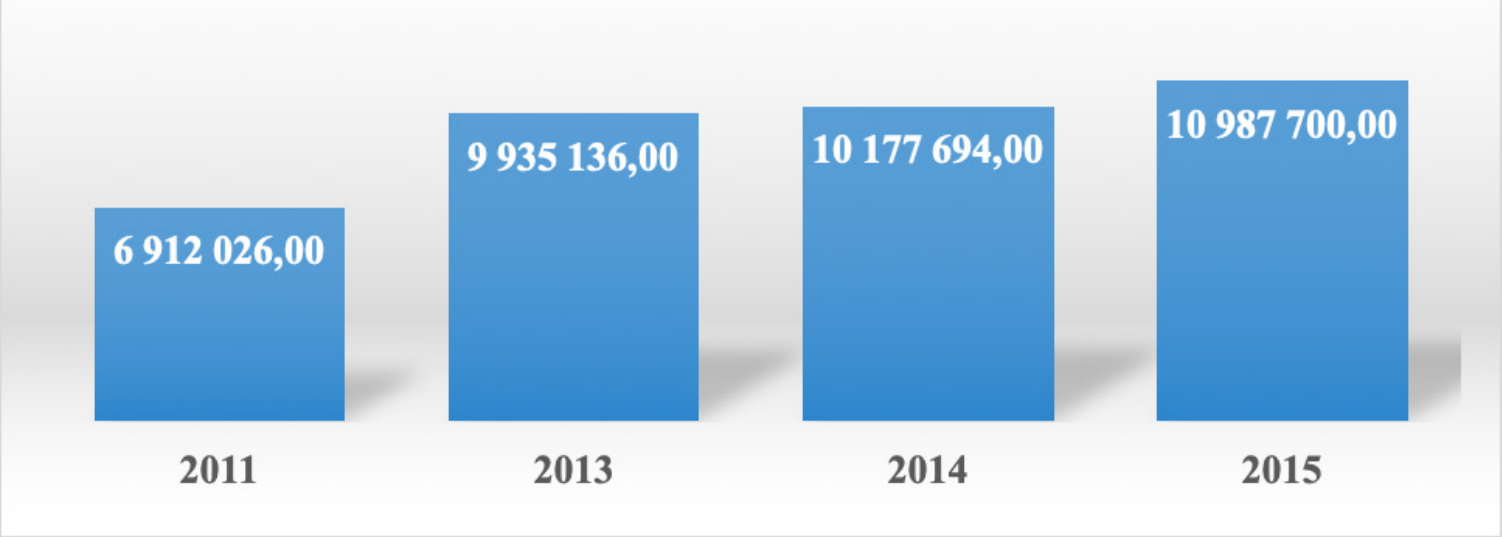

Fuente: elaboración propia basada en los datos estadísticos obtenidos del SRI (2014).

El incremento en el total de recaudación del IVA mensual en la provincia de Santa Elena, entre los años 2011 y 2015, es de un 58,96 \%, lo que demuestra el incremento en las actividades comerciales de la zona. Como se indicó anteriormente, la bonanza petrolera y la sensación de seguridad económica estimularon un incremento de los negocios y de la aportación tributaria.

Es evidente que la recaudación tributaria ha constituido la base fundamental del presupuesto público, por lo que se considera de vital importancia fortalecer el control tributario como soporte para la potencialización de la economía ecuatoriana de acuerdo con los principios de justicia y la equidad tributaria que se promulgan en la Constitución.

\section{Análisis de las multas aplicadas durante el período 2011-2015}

El informe de la Conferencia de las Naciones Unidas sobre Comercio y Desarrollo (UNCTAD, 2015) refiere que "América Latina sería la región más afectada por la evasión fiscal". Godoy (2015) reconoce también en su investigación que "Ecuador es uno de los países que mayor evasión tributaria registra en la región", afirmación que fundamenta en los problemas estructurales del país que inciden en el elevado nivel de incumplimiento por parte de los contribuyentes.

Dentro de estos destaca, principalmente, los altos costos del capital de operación, el bajo nivel de acceso a crédito bancario público y/o privado y 
la falta de empleo formal que conlleva a los individuos a la búsqueda de modos de subsistencia, destacando que "el empleo informal en Ecuador en la actualidad ocupa cerca del $50 \%$ de la Población Económicamente Activa en el país". Reconoce, además, la utilización de diversas formas de disfrazar la evasión tributaria como la utilización de viviendas como locales comerciales, concluyendo en su estudio que la población de Ecuador carece de un conocimiento tributario inherente. Para cumplimentar el análisis desarrollado hasta el momento, y por su relación con la evasión fiscal, se procede a realizar un análisis de las contravenciones detectadas en el período de estudio.

De acuerdo al análisis de las multas durante el período 2011-2015 (mostrado en la Tabla 3), se observa un incremento del monto recaudado por las sanciones aplicadas con una ligera variación a la disminución hacia el final del período. Teniendo en cuenta el monto de la recaudación, se puede observar que el grado de representatividad tiene muy poca variación, siendo en el 2011 el 2,76 \% de lo recaudado, en el 2012 el 3,34\%, en el 2013 el $3,27 \%$, en el 2014 el 3,84 \% y en el 2015 el $2 \%$.

Tabla 3.

Análisis de las variaciones anuales en la recaudación y las multas aplicadas.

\begin{tabular}{|c|c|c|c|c|}
\hline AÑOS & RECAUDACIÓN & VARTACIÓN & MULTAS & VARIACIÓN \\
\hline 2011 & 16885740,45 & & 466856,60 & \\
\hline 2012 & 19255763,56 & $14,04 \%$ & 643315,62 & $37,80 \%$ \\
\hline 2013 & 24206309,05 & $25,71 \%$ & 790449,13 & $22,87 \%$ \\
\hline 2014 & 24929111,24 & $2,99 \%$ & 958261,85 & $21,23 \%$ \\
\hline 2015 & 24674949,00 & $-1,02 \%$ & 494248,00 & $-48,42 \%$ \\
\hline
\end{tabular}

Fuente: elaboración propia basada en los datos estadísticos obtenidos del SRI (2014).

Asimismo, se puede observar en la Figura 3 que las contravenciones y faltas reglamentarias representan más del $50 \%$ del monto anual, siendo los años 2014 y 2015 los que muestran los mayores valores.

Sin embargo, al analizar la estructura de las sanciones aplicadas durante el período 2011-2015, por su clasificación tal y como se muestra en la Tabla 4, se puede apreciar que el crecimiento de la clasificación "contravenciones y faltas reglamentarias" ha sido generado principalmente por el concepto "multas tributarias", que mantuvo un valor superior en los años 2014 y 2015. Las principales causas se corresponden con la no emisión de comprobantes de ventas por operación mercantil, con emitir facturas caducadas o sin cumplimentar los requisitos exigidos por la ley, con permitir el uso del registro de inscripción (RUC) por parte de terceros y con ocultar la existencia de otros establecimientos de los que es propietario el contribuyente.

Asimismo, la clasificación "Declaraciones Tardías" muestra valores significativos fundamentados principalmente por el concepto "Interés por Mora Tributaria" que, ante una reducción en el 2014, retoma el alza en el 2015. Las causas principales están relacionadas con incumplimientos en la presentación de la información requerida oportunamente. 
Figura 3.

Análisis porcentual del comportamiento de las sanciones aplicadas durante el período 2011-2015 en la provincia de Santa Elena.

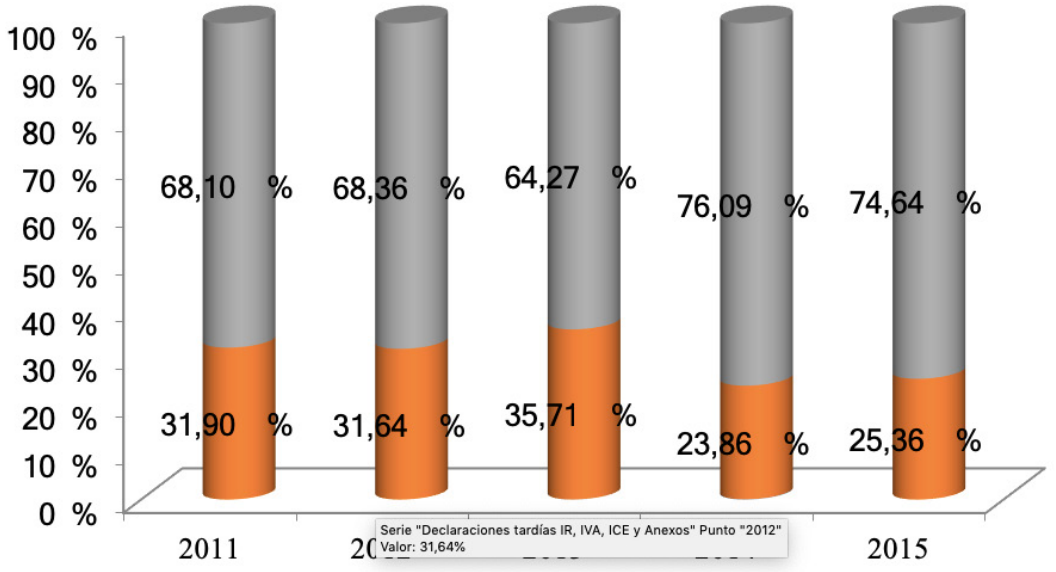

Contravenciones y faltas
reglamentarias

- Declaraciones tardías IR, IVA, ICE y Anexos

Falta de presentación o presentación tardía de declaraciones patrimoniales

Fuente: elaboración basada en los datos estadísticos obtenidos del SRI (2014).

\section{Tabla 4.}

Datos de las sanciones aplicadas durante el periodo 2011-2015 en la provincia de Santa Elena.

\begin{tabular}{|c|c|c|c|c|c|c|}
\hline $\begin{array}{l}\text { CLASIFICACIÓN } \\
\text { INFRACCIONES }\end{array}$ & DESCRIPCIÓN & 2011 & 2012 & 2013 & 2014 & 2015 \\
\hline $\begin{array}{l}\text { Falta de } \\
\text { presentación o } \\
\text { presentación tardía } \\
\text { de declaraciones } \\
\text { patrimoniales }\end{array}$ & $\begin{array}{l}\text { Multa por } \\
\text { Presentación } \\
\text { Tardía de } \\
\text { Declaraciones } \\
\text { Patrimoniales }\end{array}$ & & & 158,06 & 47,91 & \\
\hline \multirow{4}{*}{$\begin{array}{c}\text { Declaraciones } \\
\text { tardías IR, IVA, ICE } \\
\text { y Anexos }\end{array}$} & $\begin{array}{c}\text { Multa por } \\
\text { Presentación } \\
\text { Tardía de Anexos }\end{array}$ & & & 16596,28 & 4149,30 & 444,82 \\
\hline & $\begin{array}{l}\text { Multa Anexos } \\
\text { Declaraciones } \\
\text { Impuesto Renta }\end{array}$ & & & & & 345,97 \\
\hline & $\begin{array}{c}\text { Multa Anexos } \\
\text { Declaraciones IVA }\end{array}$ & & & & & \\
\hline & $\begin{array}{c}\text { Interés por Mora } \\
\text { Tributaria }\end{array}$ & 148927,26 & 203545,06 & 265619,54 & 18709,23 & 124599,92 \\
\hline
\end{tabular}


La conciencia tributaria en el proceso de recaudación de impuestos en Santa Elena

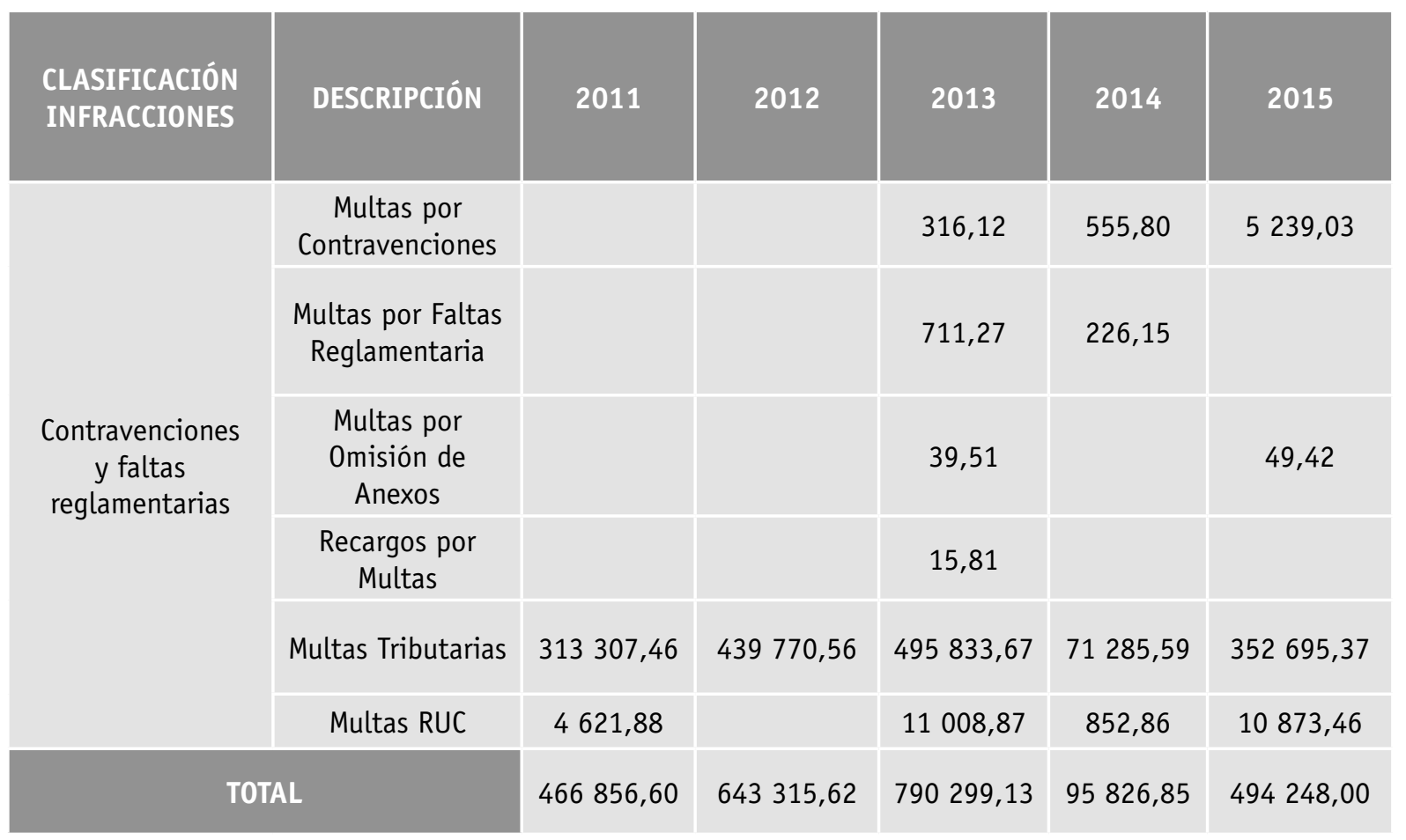

Fuente: elaboración basada en los datos estadísticos obtenidos del SRI (2014).

Teniendo en cuenta el análisis realizado, y dado que el aumento de la recaudación en el período estuvo fundamentado en un aumento de la productividad, se suscita un análisis de las acciones de capacitación.

\section{Evaluación del alcance del conocimiento tributario de los pobladores de la provincia de Santa Elena}

Para conocer la realidad referente al conocimiento tributario de los habitantes de la provincia de Santa Elena, se aplicó una encuesta a 68 habitantes del lugar; esta, posteriormente tabulada, mostró los resultados siguientes:

El $58 \%$ de los encuestados conoce el significado de las siglas "SRI" como del Servicio de Rentas Internas, a pesar de que no tienen definida de forma clara las atribuciones y funciones de esta entidad; por su parte, un $48 \%$ de los encuestados desconoce las siglas y eligió otras opciones irreales que le fueron planteadas.

Respecto al alcance o definición del impuesto a la renta, solo un $4 \%$ de los encuestados tiene clara la idea del impuesto; sin embargo, el $64 \%$ confunde el impuesto a la renta (IR) con el impuesto al valor agregado (IVA).

En lo que se refiere a la capacitación tributaria, un $88 \%$ de los encuestados indica no haber recibido nunca este tipo de capacitaciones, por lo que justifica su bajo conocimiento; solo un $4 \%$ de los encuestados señala haber recibido algún tipo de preparación en cierto momento, pero aclara que fue de forma autodidacta.

Respecto al destino de las recaudaciones, el $44 \%$ de la población de Santa Elena cree que lo recaudado por concepto de impuestos no es invertido en infraestructura ni servicios para la provincia; considera que todos esos recursos se inviertan en otras localidades o tienen un destino desconocido.

El $62 \%$ de los encuestados reconoce no solicitar facturas cuando efectúa alguna transacción mercantil o comercial, lo que denota falta de conocimiento 
tributario en la zona; por esta razón se planteó la pregunta sobre la existencia de una conciencia tributaria en Santa Elena: un $72 \%$ de los encuestados respondió a las opciones "muy pocas" o "no existe", mientras que un $22 \%$ no respondió a la interrogante y solo un $6 \%$ respondió que tiene buena conciencia tributaria.

Como resultado de los datos obtenidos en la aplicación de las encuestas, se demuestra que, de forma generalizada, existe falta de conocimiento y capacitación en los contribuyentes y ciudadanos en general, en lo concerniente a sus obligaciones tributarias.

\section{DISCUSIÓN}

Se ha experimentado un crecimiento del monto recaudado por las sanciones aplicadas durante el 2011-2015 en la provincia de Santa Elena, lo que origina un análisis acerca de la efectividad de las acciones de capacitación que se han venido generando por el Servicio de Rentas Internas en esta localidad. Se evidencia, además, un alto grado de desconocimiento en materia tributaria en los pobladores del lugar, por lo que proveer a los ciudadanos de una conciencia tributaria a partir de la formación de facilitadores desde los gremios existentes en la localidad permitirá adquirir conocimientos y valores que modifiquen su forma de pensar, obrar y concebir los tributos; esta debe ser la estrategia principal del Servicio de Rentas Internas.

La revista Fiscalidad del Servicio de Rentas Internas (2013) plantea que "la construcción de ciudadanías activas, políticamente claras, en medio de la participación en la toma de decisiones requiere de propuestas de aprendizaje orientadas a la consecución de autonomías y derroteros libremente elegidos" (Centro de Estudios Fiscales, 2013, p. 22).

Se tiene conocimiento de que una de las fortalezas de la localidad son los gremios $\mathrm{y} / 0$ asociaciones que posee; en este sentido, se propone diseñar una guía metodológica que permita elaborar programas de capacitación en materia tributaria para los ciudadanos de la provincia de Santa Elena a partir de la formación de facilitadores en los gremios y asociaciones del lugar.

Los ejes centrales para el desarrollo de una conciencia tributaria son la formación y el desarrollo de la conciencia ciudadana y el desarrollo de una guía metodológica que permita diseñar programas de capacitación en materia tributaria para los ciudadanos de la provincia de Santa Elena. Las falencias detectadas en el estudio realizado permitirán dirigir los esfuerzos hacia la acción deseable de capacitación con el concurso de los gremios y las organizaciones de la localidad, facilitará el acceso a todos los lugares de la provincia y ayudará a que se comparta información y se confronte entre todos, trabajando para encontrar soluciones comunes sobre la idea de que se estarían formando facilitadores múltiples.

Con la participación ciudadana y comunitaria se busca lograr un mejoramiento en la toma de decisiones de los aspectos y problemas tributarios que se encuentran en la comunidad. Los cursos estarían insertados dentro del Plan de Capacitación que el Servicio de Rentas Internas planifica y presupuesta cada año por las regiones del país, siendo una propuesta a realizar a la entidad reguladora.

De acuerdo con De Kereki (2003), para que la acción de capacitación tenga el efecto que se espera se deben determinar ciertos criterios de selección, tales como:

- Nivel Educacional.

- Interés y compromiso con la temática.

- Compromiso con la actividad de replicación

- Conocimientos generales mínimos de género y economía.

- Acceso a herramientas informáticas y de comunicación (p. 95).

Se considera imprescindible la planificación organizada de toda actividad cognoscitiva para que el proceso sea sostenible en el tiempo. El objetivo formativo para el desarrollo de la actividad debe relacionarse con la apropiación por 
parte de los formadores de los diversos aspectos exigidos por la legislación ecuatoriana vigente en materia tributaria.

El curso de formación puede contar con dos módulos: un primer módulo introductorio, a través del cual los formadores incorporen contenidos didácticos y pedagógicos que permitan posteriormente replicar la actividad, y un segundo módulo, considerado el específico, relacionado con los principios fundamentales que se requieren para realizar las declaraciones de impuestos y las implicaciones de los incumplimientos. Este último será el de mayor exigencia, siendo solo posible su comprensión una vez vencido el primer módulo. Las clases se desarrollarán mediante dos formas: la exposición de los temas por instructores previamente capacitados y seminarios elaborados por las personas participantes.

\section{CONCLUSIÓN}

Debido al aumento de actividades comerciales y turísticas, que derivaron en mayores transacciones y que se convirtieron en hechos generadores de impuestos, las recaudaciones tributarias en la provincia de Santa Elena reportaron un aumento en el período 2011-2013 asociado al incremento de la productividad; esto significa que no hubo un incremento importante en el número de contribuyentes. Es importante destacar que, durante los años 20142015, se generó un decrecimiento en los montos de recaudación relacionados con afectaciones de tipo socio-económico como la inflación, el desempleo, el subempleo, etc., derivadas de la coyuntura económica experimentada por el país tras la caída del precio del petróleo y la apreciación del dólar.

El monto recaudado por las sanciones aplicadas mantuvo una tendencia al aumento; adicionalmente, se encuentra el nivel de preparación de la ciudadanía del lugar en materia tributaria que se obtuvo mediante una encuesta que arrojó resultados negativos: resultados que promueven un análisis de las acciones de instrucción que han sido ofrecidas por el Servicio de Rentas Internas y que conllevan a aseverar que la capacitación tributaria no llega al ciudadano común debido a que las capacitaciones in situ se realizan solamente en los principales cantones de las provincias del Ecuador.

La propuesta de elaboración de una guía metodológica para crear formadores en materia de tributación en asociación con gremios de la localidad es una invitación a la participación de la ciudadanía para lograr un mejoramiento real y efectivo a corto plazo en la toma de decisiones de los aspectos y problemas que se encuentran en la comunidad y a contribuir a la formación y al desarrollo de la conciencia tributaria ciudadana.

Se recomienda la adecuación de oficinas temporales/ móviles en comunas y sectores alejados y de difícil acceso de la provincia con el objetivo de incrementar el número de contribuyentes y persuadir respecto al pago de las obligaciones tributarias.

En situaciones de aparente crisis económica, recesión económica o desaceleración de la economía local por eventos externos es prudente evaluar los porcentajes y la periodicidad de pagos de impuestos, ya que en tiempos críticos los pagos del contribuyente están sujetos a factores de tipo personal.

\section{REFERENCIAS BIBLIOGRÁFICAS}

Andino A. M., Arias U. D., Carrasco, V. C. M., Carrillo, M. P., Carpio, R. R., Chiliquinga, C. D., ... Torres E. A. (2012). Una Nueva Política Fiscal para el Buen Vivir: La equidad como soporte del pacto fiscal. Quito, Ecuador: Ediciones Abya-Yala.

Balseca, M. M. R. (2012). Equidad y Desarrollo. Quito, Ecuador: SRI.

Betancur, A. J. H. (2010). La ética de la responsabilidad social empresarial. Katharsis, 9, 73-92. https://doi.org/ https://doi.org/10.25057/issn.2500-5731

Cabezas, J. M. E. (2010). Analisis de los efectos economicos en los impuestos en el Ecuador (Tesis de Diplomado). Universidad Técnica Particular de Loja, Loja, Ecuador.

Castañeda, R. V. M. (2015). La moral tributaria en América Latina y la corrupción como uno de sus determinantes. Revista Mexicana de Ciencias Politicas y Sociales, 60(224), 103-132. https://doi.org/10.1016/ S0185-1918(15)30005-2 
Centro de Estudios Fiscales. (2013). Elementos socio-políticos y éticos de las ciudadanías y de la ciudadanía fiscal. Fiscalidad, VII, 8-50.

Conferencia de las Naciones Unidas sobre Comercio y Desarrollo - UNCTAD. (2015). Informe sobre el comercio y el desarrollo 2015. Nueva York y Ginebra: Naciones Unidas. Recuperado de http://unctad.org/es/ PublicationsLibrary/tdr2015_es.pdf

Constitución de la República del Ecuador. (2008). Registro Oficial Organo del Gobierno de Ecuador (No.449). Recuperado de https://www.corteconstitucional.gob. ec/images/contenidos/quienes-somos/Constitucion_ politica.pdf

De Kereki, G. I. F. (2003). Modelo para la creación de entornos de aprendizaje basados en técnicas de gestión del conocimiento (Tesis Doctoral). Recuperado de http:// www.ort.edu.uy/fi/pdf/Tesis.pdf

Del Busto Vargas, J. (2014). Etica Tributaria. TH MISRevista de Derecho, 12, 10-15. Recuperado de http://revistas.pucp.edu.pe/index.php/ themis/article/viewFile/9295/9710

Fascioli, A. (2010). Ética del cuidado y ética de la justicia en la teoría moral de Carol Gilligan. ACTIO, (12), 41-57. Recuperado de http://www.lappu. edu.uy/ActioSite09/Textos/12/Fascioli12.pdf

Godoy Peralta, M. G. (2015). Desarrollo de la Cultura Tributaria en el Ecuador y su Impacto en la Recaudación Tributaria. Periodo: 2011-2014 (tesis de maestría). Recuperado de http://repositorio. ug.edu.ec/bitstream/redug/7868/1/IVH-DR. GODOY PERALTA-AGOSTO 7 -2015.pdf

Instituto Nacional de Estadísticas y Censo - INEC. (2010). Fascículo Provincial Santa Elena. Recuperado de http://190.152.152.74//wp-content/descargas/Manu-Lateral/Resultados-provinciales/ santa_elena.pdf

Jaramillo, G. B. K. y Aucanshala, N. L. A. (2013). Optimización de la Gestión de Recaudación Impuestos Seccionales Aplicado en el Ilustre Municipio de Riobamba. Recuperado de http:// www.dspace.espol.edu.ec/xmlui/bitstream/ handle/123456789/30334/D-P11782. pdf? sequence $=-1$ \&isAllowed $=y$

Jiménez, J. (2015). El sistema tributario de Montecristi: teoría y praxis. En A. S. (Coordinador). (Ed.), La Nueva Economía en la Nueva Constitución del Ecuador (p. 115-141). Quito: SRI - CEFI.

La recaudación se triplicó entre 2007 y 2013. (16 de diciembre de 2014). El Telégrafo, p. 1. Recuperado de http:// www.eltelegrafo.com.ec/noticias/economia/8/ la-recaudacion-se-triplico-entre-2007-y-2013
Leon, M. I., Martinez, J., Zavala, A. L. y Espinel, R. (2009). Propuestas de política social para erradicar la pobreza en las comunidades de la península de Santa Elena (tesis de pregrado). Recuperado de http://www.dspace.espol.edu. ec/handle/123456789/461

Ley de Creacion del Servicio de Rentas Internas (Ley No. 41) (Registro Oficial No. 206 ) (1997).

Lozano, E. y Tamayo, D. (2016). Gestión de la ética en la administración tributaria colombiana. Revista de Derecho Privado, (55), 36. https:// doi.org/D0I: http://dx.doi.org/10.15425/ redepriv.55.2016.09

Maldonado, 0. B. V. (2009). La capacidad contributiva como objetivo para investigar el fundamento del derecho del Estado, a incorporar a la legislación tributaria un determinado tributo (tesis de pregrado). Universidad Técnica Particular de Loja, Ecuador. Recuperado de http://dspace.utpl.edu.ec/ handle/123456789/1402

Moreira, C. M. K. (2015). Estrategia para incrementar el registro de los contribuyentes en el RUC y el RISE en el cantón Jipijapa, año 2014 (tesis de maestría). Recuperado de http://repositorio.ug.edu.ec/ handle/redug/10118

Novoa, H. G. (2006). El Principio de la Capacidad Contributiva. Revista Derecho y Sociedad, 27. Recuperado de http://revistas.pucp.edu. pe/index.php/derechoysociedad/article/ view/17169/17458

Peláez F. M. J. y Gutiérrez, J. N. D. (2016). Los tributos y su aporte al Presupuesto General del Estado un análisis comparativo en la República del Ecuador: períodos 2013-2014-2015. Revista Observatorio de la Economía Latinoamericana, Ecuador, p. 1-13. Recuperado de http://www.eumed.net/cursecon/ecolat/ec/2016/tributos.html

Reglamento para la aplicación de la Ley del Régimen Tributario Interno (Decreto No. 580)(Registro Oficial No.448) (2015).

Sarmiento, P. A. E. (2015). La prescripciòn como modo de extinguir las obligaciones tributarias dentro de la legislación ecuatoriana (Tesis de Pregrado). Universidad de Cuenca. Recuperado a partir de http://dspace. ucuenca.edu.ec/bitstream/123456789/23289/1/ Monografía.pdf

Servicio de Rentas Internas - SRI. (2014). Instructivo para la aplicación de Sanciones Pecuniarias. Quito: Editorial del Servicio de Rentas Internas.

Valdivia, B. A. E. (2010). El Sistema Tributario. Revista Observatorio de la Economía Latinoamericana, Ecuador, 140. Recuperado de http://www.eumed. net/cursecon/ecolat/cu/2010/aevb2.htm 
Vargas, R. J. C. (2014). Ciudadanía fiscal en la formación por competencias. Fiscalidad, (3), 74-106.

Vicente de la Casa, F. (2012). Los principios de capacidad económica y no confiscatoriedad como límite a la concurrencia de tributos. Crónica Tributaria, 2012(144), 1-27.

Washco Castro, T. L. (2015). Análisis de las reformas tributarias e incidencia en la recaudación de los principales impuestos del Ecuador, 2009- 2013 (tesis de maestría). Universidad de Cuenca, Ecuador. Recuperado de http://dspace.ucuenca.edu.ec/ bitstream/123456789/21566/1/tesis.pdf
Yañez, J. (2015). Evasión Tributaria: Atentado a La Equidad. Revista de Estudios Tributarios Universidad de Chile, (13), 171-206. Recuperado de https:// revistaestudiostributarios.uchile.cl/index.php/RET/ article/view/39874/41444

Ziccardi, H. (2016). La ética y los impuestos. Revista San Gabriel, 1-5 . Recuperado de http://revista.colegiosangabriel.edu.ar/index.php/2016/04/07/ la-etica-y-los-impuestos/ 